\title{
Interphase cytogenetics: At the interface of genetics and morphology
}

\author{
B. Michael Ghadimi ${ }^{\mathrm{a}, *}$, \\ Kerstin Heselmeyer-Haddad ${ }^{\mathrm{a}}$, Gert Auer ${ }^{\mathrm{b}}$ and \\ Thomas Ried ${ }^{\text {a }}$ \\ ${ }^{a}$ Genetics Department, National Cancer Institute, \\ National Institutes of Health, Bethesda, MD, USA \\ ${ }^{\mathrm{b}}$ Division of Cellular and Molecular Analysis, \\ Department of Oncology and Pathology, Karolinska \\ Institute and Hospital, Stockholm, Sweden
}

Cancer is a genetic disease caused by chromosomal aberrations and gene mutations, which propel normal cells into uncontrolled growth, invasiveness and metastases formation. Because chromosomal and genetic alterations impact tumour aggressiveness, therapy response, and prognosis their detection in clinical samples (e.g., fine needle aspirates and bioptic material) plays an increasingly critical role in individualized disease management. In situ hybridization with genetic markers to tumour specimen, a concept coined "Interphase Cytogenetics" [3] allows one to visualize chromosomal alterations in intact cell nuclei. Both numerical and structural chromosomal aberrations can be visualized using suitable probes or probe cocktails. The growing knowledge on tumour specific genetic and chromosomal aberrations can therefore be translated to diagnostic pathology. The identification and validation of such biomarkers will be greatly accelerated by highthroughput analysis tools such as cDNA-arrays for the analysis of differentially expressed messages [5] and tissues arrays [14] for the simultaneous screening of the expression pattern of hundreds of different tumours (see Fig. 1).

The detection of chromosome and gene alterations in interphase nuclei of cytological specimens and tis-

\footnotetext{
*Corresponding author: B. Michael Ghadimi, National Cancer Institute/NIH, Bldg. 49, Rm. 4C24, 49 Convent Drive, Bethesda, MD 20892, USA. Tel.: (301) 402 2008; Fax: (301) 402 1204; E-mail: ghadimim@mail.nih.gov.
}

sue sections is the only technique available for the simultaneous visualization of genetic changes and cell morphology on a single cell level. This can be extended to the simultaneous assessment of immunophenotype and genotype $[2,13,22]$. The maintenance of morphological information is arguably beneficial, because the wealth of genetic and non-genetic changes that contribute to a particular phenotype is not lost. Interphase cytogenetics can be performed on freshly frozen or fixed and archived tissues and is therefore applicable to the prospective, and particularly the retrospective study of tumour tissues. The development of multiple color fluorescent as well as colorimetric detection systems affords the possibility of enumerating multiple genetic and chromosomal target regions in a single experiment [19-21].

The relevance of the "loss of inhibiting chromosomes" or the "gain of promoting chromosomes" was established almost a century ago by von Hansemann [10] and Boveri [1]. Refinement of techniques in cytogenetics and molecular cytogenetics has resulted in a resurrection of the prophetic hypothesis of these two contemporaries and large scale analysis of solid tumours through the use of comparative genomic hybridization (CGH) have revealed a surprisingly recurrent and tumour specific pattern of genomic imbalances in cancer cells (reviewed in $[6,12,18]$ ). CGH experiments require only genomic DNA extracted from formalin fixed tissue blocks and histologically characterized tissue sections and therefore allow the correlation between the cytogenetic profile and tumour phenotype and tumour stages. The application of CGH established a non-random sequence of chromosomal abnormalities during carcinogenesis $[11,16,17]$. In cervical cancer, for example, the gain of an additional copy of chromosome $3 \mathrm{q}$ occurs at the transition of preinvasive dysplastic lesions to invasive carcinomas and precedes the acquisition of a more complex pattern of DNA gains and losses at more advanced tumour stages. 


\section{Discovery}

Molecular cytogenetics (CGH, SKY)

Molecular biology (CDNA array, etc.)

Validation

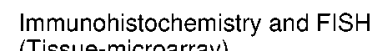

Validation

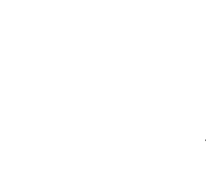

Biomarkers (DNA-probes, Antibodies) on cytological and histological preparations

Fig. 1. Flow chart: from gene discovery to clinical application.

Such a chromosomal marker has the potential to become useful as a predictor for the progressive potential of premalignant lesions. Unlike cervical carcinogenesis, a higher number of recurring chromosomal changes can be detected in tumours of, e.g., the breast, colon, ovary, and pancreas. CGH studies, however, indicate that the utilization of up to 4 different DNA probes specific for recurrently involved chromosome arms will be sufficient to detect almost all tumours. The tumour type dependent distribution of chromosomal imbalances might also assist in the differential diagnosis in cases where the site of origin cannot be unambiguously established. For example, an interphase probe set for the detection of cervical cancer will include tags for the chromosomes and chromosome arms $1 \mathrm{q}, 3 \mathrm{q}, 5 \mathrm{p}$ and 20 whereas its counterpart for breast cancer will contain probes for 1q, 4q, 6q and 8q.

The translational application of these results to the histo- and cytomorphology based diagnosis of cancer holds great promise for more than an incremental increase in diagnostics accuracy. The considerable potential of interphase cytogenetics can be deduced from the need for an early detection of malignant disease when a curative treatment is sought. Phenotypic changes in pre-invasive or early invasive disease are often less clear than those in advanced disease. DNA based assays, such as PCR, for the detection of cancer specific gene mutations are more ambiguous in early

lesions since the contamination with normal cells complicates data interpretation.

Two examples that provide diagnostic dilemmas for different reasons are breast and pancreatic carcinomas. Successful mammography screening programs in particular in Northern European countries result in an earlier detection of suspicious breast lesions and consequently in tumours that are smaller at the time of diagnosis [9]. For the cytopathologist, however, smaller tumours, more of which are naturally premalignant lesions are more difficult to definitively diagnose. It is conceivable that the complementation of morphology with pertinent genetic and chromosomal markers may fill a bothersome gap. Another tumour type whose mortality has certainly not been reduced by improved screening programs is pancreatic adenocarcinoma. In pancreatic cancer, early diagnosis is the exception, not the rule. The majority of patients die within one year after diagnosis. The application of an interphase cytogenetics based diagnostic procedure to aspiration biopsies and pancreatic juice acquired during endoscopic procedure of high risk patients are therefore a worthwhile endeavor $[7,8]$.

In general, two different kinds of probe sets are being used for interphase cytogenetics. The most frequently used probes are directed against chromosomespecific centromeric repeat units. These centromerespecific probes are easy to use, have a superb signal to noise ratio, and their ability to enumerate chromosome copy number changes have been amply demonstrated in a variety of tumours (for a review see [4]). Their most relevant limitation is that although copy number changes involving entire chromosomes (including the centromere) can be visualized, chromosome arm changes remain undetected. CGH analyses, however, have revealed abundant evidence that imbalances of chromosomal arms occur frequently in solid tumours. Hence, the usefulness of interphase cytogenetics would be increased through the use of locus- and gene-specific probes targeting highly recurrent, tumour type- and tumour stage-specific aberrations. A panel of differently labeled gene-specific probes would not only help in the crucial decision whether a tumourous lesion is benign or malignant, but would also allow the investigator to simultaneously assess the deletion and amplification status of pertinent tumour suppressor genes or oncogenes, respectively. The importance of determining such a genetic status for prognostication and choice of therapeutic regimen cannot be overestimated and will be part of a molecular profiling of cancer cells that is likely to become a prerequisite for an individualized 
therapy plan. An example is the prognostic and therapeutic relevance of the erbB2 status in patients with breast cancer (for a review see [15]). Only patients with erbB2 gene amplification might benefit from an adjuvant or palliative treatment with Herceptin, a monoclonal antibody directed against the erbB2 epitope.

$\mathrm{BAC}$ or $\mathrm{P} 1$ clones are the preferred vector if single gene or locus specific probes are required because of their size (approximately $100 \mathrm{Kbp}$ ) and vector to insert ratio. Compared to YAC clones, the frequency of chimarism is low. The hybridization efficiency and signal to noise ratio can be further optimized when single clones are extended to small contigs. A centralized BAC clone repository that covers the entire human genome with a cytogenetically linked resolution of about $1 \mathrm{Mbp}$ is presently assembled. This Cancer Chromosome Aberration Project (C-cap) is part of the Cancer Genome Anatomy Project funded through the National Cancer Institute, USA. The progress of this clone mapping project and additional pertinent information can be obtained from the C-cap website (http://www.ncbi.nlm.nih.gov/CCAP/). C-cap's goal is to integrate the physical, genetic, and cytogenetic maps of the human genome and secure ready access to $\mathrm{BAC}$ clones from any given chromosomal location and clones that are specific for major tumour suppressor genes and oncogenes.

The future of interphase cytogenetics looks promising, however, its introduction into the clinical setting requires further improvements and validation. The analysis of hundred of cells is currently performed by manually counting FISH signals in interphase nuclei. Signal acquisition, as well as quantitation, needs to be automated to allow the timely screening of clinical samples prior to the introduction into clinics. Problems of nuclei segmentation, nuclei overlaps and signal thresholds also need to be addressed before reliable scoring can be achieved. Automated spot counting requires fully automated hardware as well as sophisticated software that will allow high-throughput screening of hundreds of clinical samples. The construction of DNA-probe kits, individualized for each given tumourtype, containing up to 4 BAC clones labeled with different fluorochromes (multicolor) will enable the detection and prognostication of malignancies. It is also conceivable that different kits can be constructed for diagnostics, prognostics or therapy-monitoring.

The correlation of certain biomarkers with morphological phenotype still warrants validation for certain precursor lesions. In this respect, prospective studies, e.g., demonstrating a malignant potential of dysplastic lesions in the breast with concurrent gains of an extra copy of chromosome 1q, are urgently needed. The use of interphase cytogenetics to samples like aspiration cytologies or cytospins can be applied to any given clinical sample, including blood preparations. The isolation of circulating epithelial cells in the blood using iron particles coated with antibodies specific for epithelial markers is an especially useful technology to which interphase cytogenetics can be applied. First, the unambiguous identification of cells with or without genetic alterations will significantly contribute to the specificity of this approach. The goal is to develop and optimize this non-invasive procedure for early diagnosis of symptom-free patients belonging to either hereditary or environmental risk groups. Second, the verification of circulating cells and, moreover the detection of circulating cells with certain prognostic characteristics (e.g., erbB2), might have therapeutic consequences for individualized patient treatment. Third, this noninvasive "blood test" could accompany a patient's adjuvant or palliative treatment and might allows clinicians to monitor in a very sensitive manner responsiveness to therapy.

In conclusion, interphase cytogenetics has the potential to become a powerful tool for early and improved cancer diagnosis and can contribute to molecular profiling by identifying the genetic makeup of cells.

\section{Acknowledgements}

The authors wish to thank Dr M.J. Difilippantonio for critically reading the manuscript and many helpful discussions. Dr B.M. Ghadimi received a postdoctoral fellowship from the Deutsche Krebshilfe.

\section{References}

[1] T. Boveri, Zur Frage der Entstehung maligner Tumoren, Fischer Verlag, Jena, 1914.

[2] J. Bulten, P.J. Poddighe, J.C. Robben, J.H. Gemmink, P.C. de Wilde and A.G. Hanselaar, Interphase cytogenetic analysis of cervical intraepithelial neoplasia, Am. J. Pathol. 152(2) (1998), 495-503.

[3] T. Cremer, J. Landegent, A. Bruckner, H.P. Scholl, M. Schardin, H.D. Hager, P. Devilee, P. Pearson and M. van der Ploeg, Detection of chromosome aberrations in the human interphase nucleus by visualization of specific target DNAs with radioactive and non-radioactive in situ hybridization techniques: diagnosis of trisomy 18 with probe L1.84, Hum. Genet. 74(4) (1986), 346-352. 
[4] H. van Dekken, C. Rosenberg, P.J. Krijtenburg and J.C. Alers, Interphase cytogenetics and comparative genomic hybridization of human epithelial cancers and precursor lesions, Histochem. Cell. Biol. 108(4-5) (1997), 419-430.

[5] D.J. Duggan, M. Bittner, Y. Chen, P. Meltzer and J.M. Trent, Expression profiling using cDNA microarrays, Nat. Genet. 21(1) (1999), 10-14.

[6] F. Forozan, R. Karhu, J. Kononen, A. Kallioniemi and O.P. Kallioniemi, Genome screening by comparative genomic hybridization, Trends Genet. 13(10) (1997), 405-409.

[7] S. Fukushige, T. Furukawa, K. Satoh, M. Sunamura, M. Kobari, M. Koizumi and A. Horii, Loss of chromosome 18q is an early event in pancreatic ductal tumorigenesis, Cancer Res. 58(19) (1998), 4222-4226.

[8] B.M. Ghadimi, E. Schröck, R.L. Walker, D. Wangsa, A. Jauho, P.S. Meltzer and T. Ried, Specific chromosomal aberrations and amplification of the AIB1 nuclear receptor coactivator gene in pancreatic carcinomas, Am. J. Pathol. 154(2) (1999), 525536.

[9] M. Hakama, E. Pukkala, M. Heikkila and M. Kallio, Effectiveness of the public health policy for breast cancer screening in Finland: population based cohort study, BMJ 22(7084) (1997), 864-867.

[10] D. von Hansemann, Über assymetrische Zelltheilung in Epithelkrebsen und deren biologische Bedeutung, Virchows Archiv (A) 119 (1890), 299-326.

[11] K. Heselmeyer, E. Schröck, S. du Manoir, H. Blegen, K. Shah, R. Steinbeck, G. Auer and T. Ried, Gain of chromosome 3q defines the transition from severe dysplasia to invasive carcinoma of the uterine cervix, Proc. Natl. Acad. Sci. USA 93 (1996), 479-484.

[12] S. Knuutila, A.M. Bjorkqvist, K. Autio, M. Tarkkanen, M. Wolf, O. Monni, J. Szymanska, M.L. Larramendy, J. Tapper, H. Pere, W. El-Rifai, S. Hemmer, V.M. Wasenius, V. Vidgren and Y. Zhu, DNA copy number amplifications in human neoplasms: review of comparative genomic hybridization studies, Am. J. Pathol. 152(5) (1998), 1107-1123.

[13] S. Knuutila, S.J. Nylund, M. Wessman and M.L. Larramendy, Analysis of genotype and phenotype on the same interphase or mitotic cell. A manual of MAC (morphology antibody chromosomes) methodology, Cancer Genet. Cytogenet. 72(1) (1994), $1-15$.
[14] J. Kononen, L. Bubendorf, A. Kallioniemi, M. Bärlund, P. Schraml, S. Leighton, J. Torhorst, M.J. Mihatsch, G. Sauter and O.P. Kallioniemi, Tissue microarrays for high-throughput molecular profiling of tumor specimens, Nat. Med. 4(7) (1998), 844-847.

[15] F. Revillion, J. Bonneterre and J.P. Peyrat, ERBB2 oncogene in human breast cancer and its clinical significance, Eur. J. Cancer 34(6) (1998), 791-808.

[16] T. Ried, K. Heselmeyer-Haddad, H. Blegen, E. Schröck and G. Auer, Genomic changes defining the genesis, progression, and malignancy potential in solid human tumors: a phenotype/genotype correlation, Genes Chromosomes Cancer 25(3) (1999), 195-204.

[17] T. Ried, R. Knutzen, R. Steinbeck, H. Blegen, E. Schröck, K. Heselmeyer, S. du Manoir and G. Auer, Comparative genomic hybridization reveals a specific pattern of chromosomal gains and losses during the genesis of colorectal tumors, Genes Chromosomes Cancer 15(4) (1996) 234-245.

[18] T. Ried, M. Liyanage, S. du Manoir, K. Heselmeyer, G. Auer, M. Macville and E. Schröck, Tumor cytogenetics revisited: comparative genomic hybridization and spectral karyotyping, J. Mol. Med. 75(11-12) (1997), 801-814.

[19] E. Schröck, S. du Manoir, T. Veldman, B. Schoell, J. Wienberg, M.A. Ferguson-Smith, Y. Ning, D.H. Ledbetter, I. BarAm, D. Soenksen, Y. Garini and T. Ried, Multicolor spectral karyotyping of human chromosomes, Science 273 (1996), 494497

[20] E.J. Speel, F.C. Ramaekers and A.H. Hopman, Cytochemical detection systems for in situ hybridization, and the combination with immunocytochemistry, "who is still afraid of red, green and blue?', Histochem. J. 27(11) (1995), 833-858.

[21] M.R. Speicher, S.G. Ballard and D.C. Ward, Karyotyping human chromosomes by combinatorial multi-fluor FISH, Nat. Genet. 12(4) (1996), 368-375.

[22] K. Weber-Matthiesen, J. Deerberg-Wittram, A. Rosenwald, M. Poetsch, W. Grote and B. Schlegelberger, Translocation $t(2 ; 5)$ is not a primary event in Hodgkin's disease. Simultaneous immunophenotyping and interphase cytogenetics, Am. J. Pathol. 149(2) (1996), 463-468. 


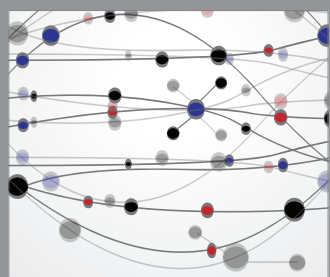

The Scientific World Journal
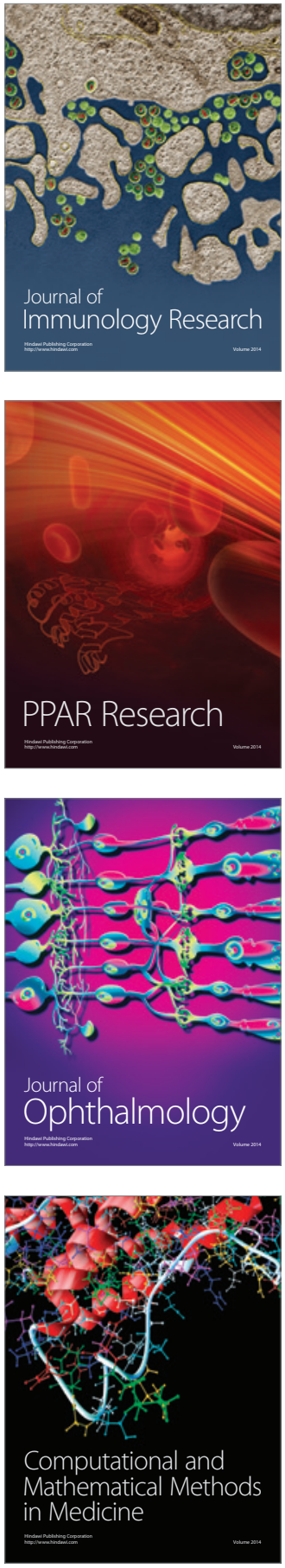

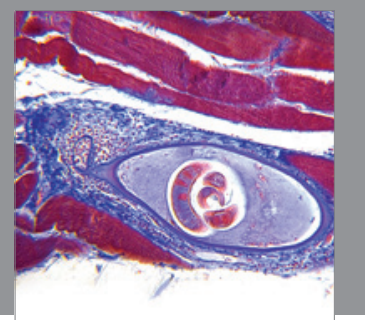

Gastroenterology

Research and Practice
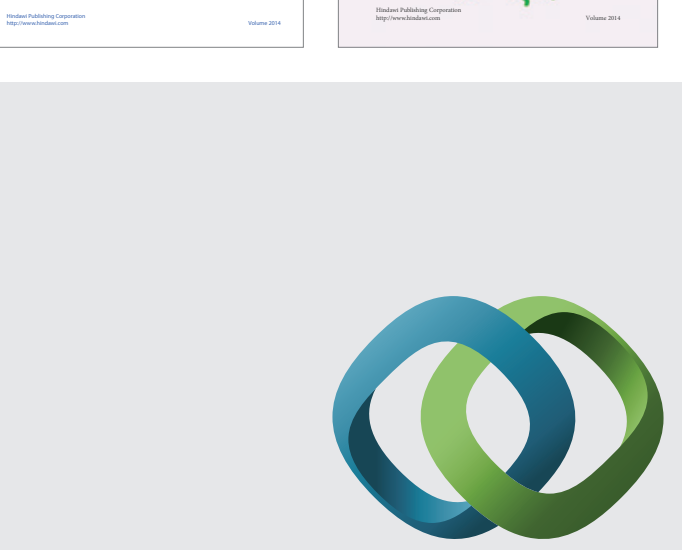

\section{Hindawi}

Submit your manuscripts at

http://www.hindawi.com
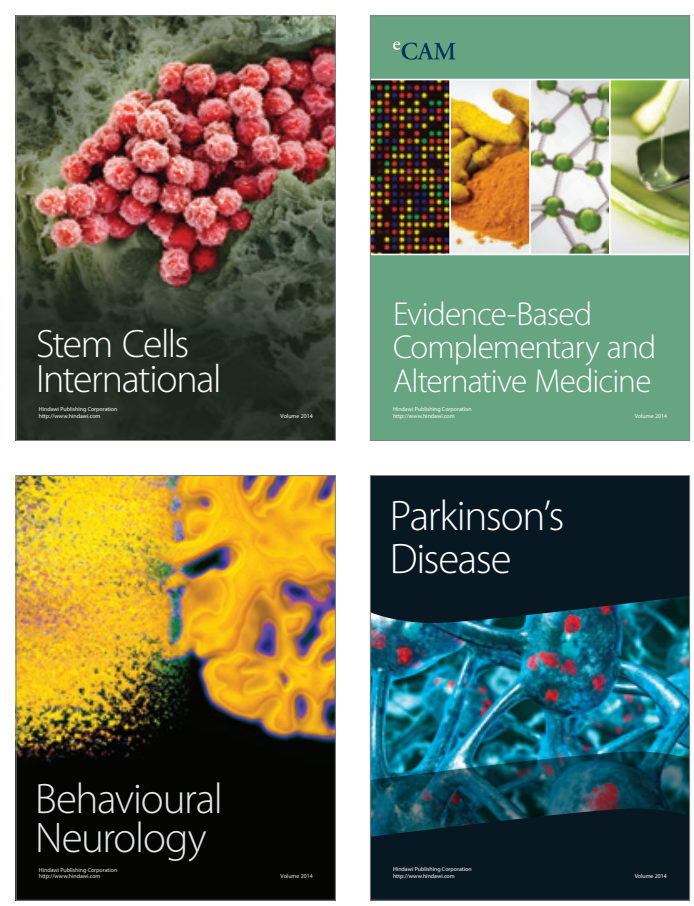

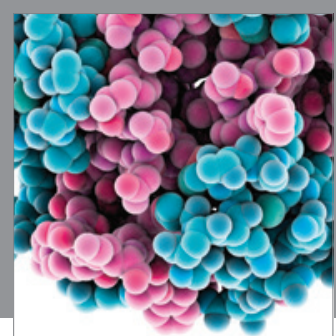

Journal of
Diabetes Research

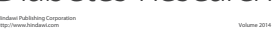

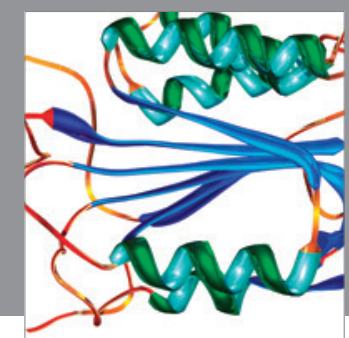

Disease Markers
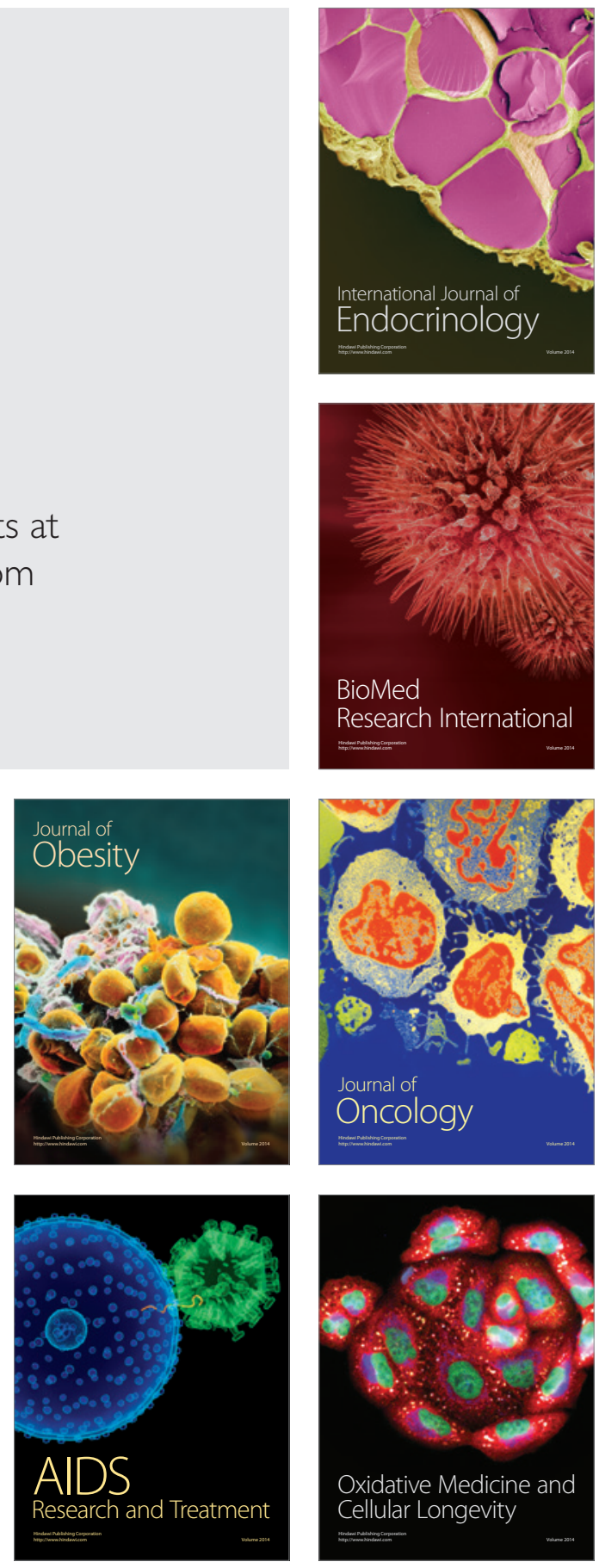